\title{
COMMUNICATION
}

\section{Perspectives de l'angiographie 3D soustractive dans les modèles de régénération oro-faciaux}

\author{
Mangione $\mathrm{F}^{1,2 *}$, EzEldeen $\mathrm{M}^{3}$, Decup $\mathrm{F}^{1,2}$, Bardet $\mathrm{C}^{1}$, Lesieur $\mathrm{J}^{1}$, Bonneau $\mathrm{M}^{4}$, Jacobs $\mathrm{R}^{3}$, Salmon \\ $\mathbf{B}^{1,2}$, Chaussain $\mathbf{C}^{1,2}$, Vital $\mathbf{S}^{1,2}$ \\ 1- EA 2496, Université Paris Descartes Sorbonne Paris Cité, Faculté de Chirurgie Dentaire, Montrouge, France \\ 2- AP-HP, Services d'Odontologie Albert Chennevier, Louis Mourier, Bretonneau and Charles Foix. Paris, France \\ 3- Department of Imaging \& Pathology/ OMFS-IMPATH Research Group KU Leuven - Group Biomedical Sciences \\ Oral and Maxillo-facial Surgery - UZ Leuven \\ 4- XP-Med, St-Germain-en-Laye, France
}

\section{Introduction}

Le potentiel de réparation tissulaire est tributaire de l'apport vasculaire et des processus de néo-angiogenèse locorégionaux. Au niveau de la sphère oro-faciale, le rôle de la composante vasculaire est capital dans les stratégies de régénération (1). Cependant, la complexité anatomique et l'hétérogénéité des tissus rendent complexe l'exploration du processus de néovascularisation par imagerie $(2,3)$.

Cette étude propose une nouvelle méthode de visualisation de la composante vasculaire crâniofaciale par angiographie 3D soustractive.

\section{Matériels et méthodes}

Des angiographies ont été pratiquées d'une part chez le rat (Lewis, 6 mois) par injection de produit de contraste iodé dans la carotide et d'autre part chez le mini-porc (FBM, 18 mois - COMITHEA 45/ INRA) par injection sélective au niveau de l'artère alvéolaire inférieure sous contrôle radiographique. La diffusion du produit de contraste a été explorée par microCT in vivo chez le rat (Quantum FX microCT, Perkin Elmer) et CT à faisceau conique en modalité soustraction chez le mini-porc (GE Discovery IGS 730). L'analyse des données a été effectuée grâce à un algorithme dédié du logiciel MeVisLab (MeVis recherche, Brême, Allemagne).

\section{Résultats}

Les acquisitions ont permis une visualisation fine et une caractérisation de la vascularisation du corps mandibulaire et de l'os alvéolaire. Après seuillage des différentes structures impliquées, une analyse quali-quantitative a été réalisée chez le mini-porc, permettant la quantification des vaisseaux sanguins et une caractérisation de leur interconnections. Les rendus volumiques du réseau vasculaire et de l'os ont également été obtenus et superposés. 


\section{Discussion}

L'angiographie par soustraction des structures anatomiques oro-faciales est un défi en raison de la forte minéralisation des tissus qui limite la sensibilité de détection de la vascularisation terminale en particulier au niveau des dents $(2,3)$.

En mettant en évidence la composante vasculaire de la région mandibulaire, cette nouvelle approche non invasive offre la possibilité de réaliser un suivi longitudinal in vivo de la néo-vascularisation, dans le cadre de la cicatrisation réparation/ régénération des tissus dentaires et oraux osseux dans des modèles précliniques.

\section{Conclusion}

Cette étude 1) démontre la faisabilité d'une visualisation et caractérisation du réseau vasculaire oro-facial grâce à l'angiographie par soustraction dans des modèles pré-cliniques et 2) offre de nouvelles perspectives pour le suivi de l'angiogenèse lors de la cicatrisation et de la régénération tissulaire.

Un développement de cette technique pourrait être impliqué dans la démarche diagnostique et thérapeutique permettant l'intégration des informations issues la composante vasculaire dans les stratégies de médecine régénérative.

\section{Références}

1) Kodonas K. et al. J Endod, 38(7):913 9.

2) Yin T. et al. Contrast Media Mol Imaging, 10(5):379-87.

3) Granata F. et al. Interv Neuroradiol, 13(4):385-8. 